\title{
High precision mass measurements of hypernuclei - achievements and perspectives
}

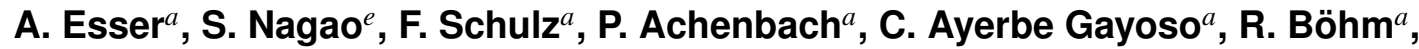 \\ O. Borodina ${ }^{a, b}$, D. Bosnar ${ }^{c}$, A. Botvina ${ }^{g}$, V. Bozkurt ${ }^{b}$, L. Debenjak ${ }^{d}$, M.O. Distler ${ }^{a}$, \\ I. Friščić ${ }^{c}$, Y. Fujii ${ }^{e}$, T. Gogami ${ }^{e}$, M. Gómez Rodríguez ${ }^{a}$, O. Hashimoto ${ }^{e *}$ S. Hirose ${ }^{e}$, \\ H. Kanda ${ }^{e}$, M. Kaneta ${ }^{e}$, E. Kim $^{b}$, Y. Kohl ${ }^{a}$, J. Kusaka ${ }^{e}$, A. Margaryan ${ }^{f}$, H. Merkel ${ }^{a}$, \\ M. Mihovilovič ${ }^{d}$, U. Müller ${ }^{a}$, S.N. Nakamura ${ }^{e}$, J. Pochodzalla ${ }^{\dagger a, g}$, C. Rappold ${ }^{b}$, \\ J. Reinhold ${ }^{h}$, T.R. Saito ${ }^{a, b, g}$, A. Sanchez Lorente ${ }^{g}$, S. Sánchez Majos ${ }^{a}$,
} B.S. Schlimme ${ }^{a}$, M. Schoth ${ }^{a}$, C. Sfienti ${ }^{a}$, S. Šrca ${ }^{d}$, L. Tang ${ }^{i}$, M. Thiel ${ }^{a}$, K. Tsukada ${ }^{e}$, A. Weber ${ }^{a}$, K. Yoshida ${ }^{b}$

A1 Collaboration

${ }^{a}$ Institut für Kernphysik, Johannes Gutenberg-Universität, 55099 Mainz, Germany

${ }^{b}$ GSI Helmholtz Centre for Heavy Ion Research, 64291 Darmstadt, Germany

${ }^{c}$ Department of Physics, University of Zagreb, 10002 Zagreb, Croatia

${ }^{d}$ University of Ljubljana and Institut "Jožef Stefan", 1000 Ljubljana, Slovenia

${ }^{e}$ Department of Physics, Tohoku University, 980-8571 Sendai, Japan

${ }^{f}$ Yerevan Physics Institute, 375036 Yerevan, Armenia

${ }^{g}$ Helmholtz Institute Mainz, 55099 Mainz, Germany

${ }^{h}$ Department of Physics, Florida International University, Miami, FL 33174, USA

${ }^{i}$ Department of Physics, Hampton University, Hampton, VA 23668, USA

E-mail: pochodza@kph.uni-mainz.de

\begin{abstract}
At the Mainz Microtron MAMI hypernuclei can be produced by $\left(\mathrm{e}, \mathrm{e}^{\prime} \mathrm{K}\right)$ reactions. A dedicated kaon spectrometer positioned at $0^{\circ}$ with respect to the incident electron beam is used to tag events involving strangeness production. By measuring the momenta of pions from two body weak decays one gains direct access to the ground state masses of the produced hyperfragments. In this contribution the motivation of the experiment and the experimental challenges are presented.
\end{abstract}

Xth Quark Confinement and the Hadron Spectrum

8-12 October 2012

TUM Campus Garching, Munich, Germany

\footnotetext{
${ }^{*}$ Deceased

† Speaker.
} 


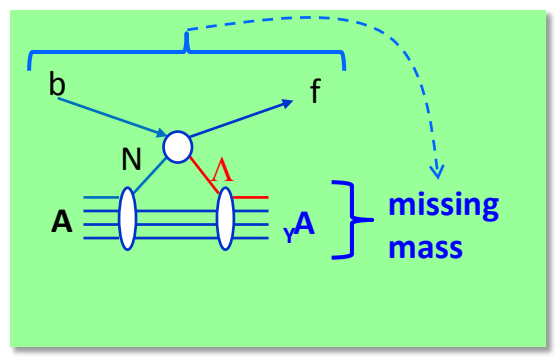

- Direct production spectroscopy

- Examples

- strangeness production $\left(\pi^{+}, \mathrm{K}^{+}\right),\left(\pi, \mathrm{K}^{0}\right)$

- strangeness exchange $\left(\mathrm{K}^{-}, \pi^{-}\right),\left(\mathrm{K}^{-}, \pi^{0}\right),\left(\mathrm{K}^{-}, \mathrm{K}^{+}\right)$

- electroproduction $\left(e, e^{\prime} K^{+}\right),\left(\gamma, K^{+}\right)$

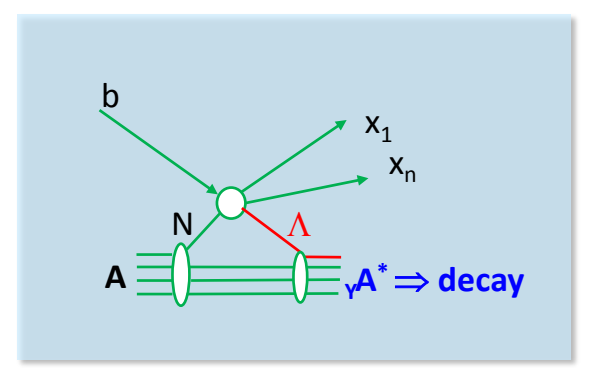

- Decay spectroscopy

- $\gamma$-decay of excited states

- $\pi$ from weak decay

- charged fragments

- Examples

- nuclear emulsions

- heavy ion reactions

- antiproton induced reactions

- continuum excitation in $\left(e, e^{\prime} K^{+}\right)$

Figure 1: Two classes of reactions to produce and study hypernuclei. Missing mass spectroscopy which employs kinematic information of the production process (left) and the decay spectroscopy which relies on the detection of the decay products (right). At MAMI hypernuclei are produced in $\left(\mathrm{e}, \mathrm{e}^{\prime} \mathrm{K}^{+}\right)$reactions. Pions from the weak decay of deexcited hyperfragments are used to identify the hypernuclei.

\section{Introduction}

The recent observation of a two-solar mass neutron star J1614-2230 [1] significantly constrains the hadronic equation of state at high densities (see e.g. [2] and references therein). However, our limited knowledge of the hyperon-nucleon, the hyperon-hyperon and the three-body YNN or YYN interactions and possible charge symmetry breaking in these interactions [3] still does not rule out the appearance of hyperons in the inner core of neutron stars $[4,5,6,7,8]$. Indeed the presence of hyperons may not only show up in the neutron star mass but can also play a crucial role in the dynamics of the core-collapse supernovae or in neutron star mergers [9]. Non-mesonic weak decays of hyperons may control the bulk viscosity of neutron star matter [10], thus regulating the e.g. r-modes instability [11] of pulsars and may leave fingerprints in the emission of gravitational waves [12]. These interrelations put the question of the matter composition of a neutron star and its fate at increasing density in focus of hypernuclear research.

The domain of light nuclei is the natural testing ground for linking nucleons and sub-nucleon degrees of freedom with complex nuclei. Combined with modern ab initio nuclear structure calculations (see e.g. [13] and references therein) this knowledge is essential to derive eventually a more general and self-consistent description of the multi-baryon-baryon interaction. Chiral effective field theory ( $\chi \mathrm{EFT}$ ) provides a link to QCD by obeying the relevant conservation laws and symmetries in the low energy-momentum domain of nuclear few-body systems. A unique feature of $\chi$ EFT is the ability to determine in an order-by-order manner a hierarchy of many-body interactions which otherwise are hardly accessible in a systematic way. Thus nucleon-nucleon interactions from $\chi \mathrm{EFT}$ 

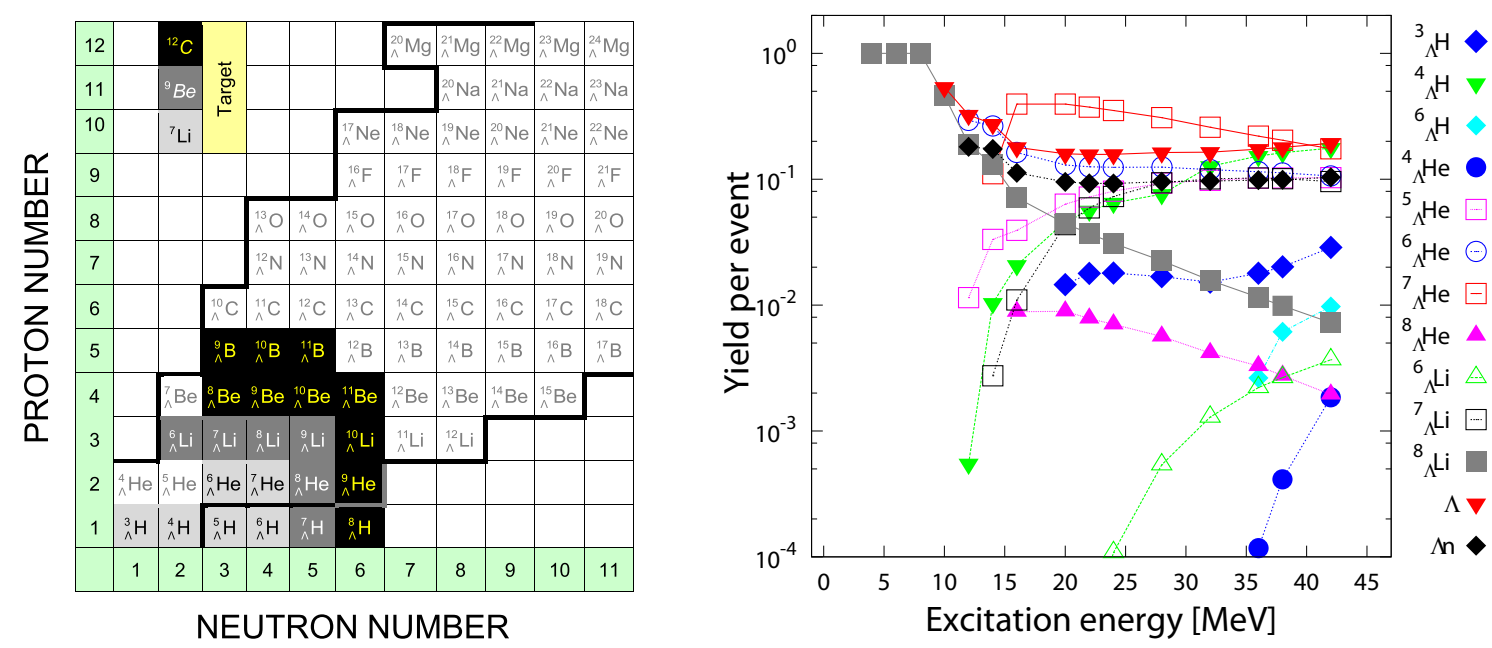

Figure 2: Left: Hypernuclei showing two-body pionic decays and which are accessible by high precision pion spectroscopy using a ${ }^{7} \mathrm{Li}$ (light grey background), ${ }^{9} \mathrm{Be}$ (grey) and ${ }^{12} \mathrm{C}$ target (black). Already with ${ }^{7} \mathrm{Li}$ and ${ }^{9} \mathrm{Be}$ targets all isobars with $\mathrm{A}=6$ can be studied. Right: Relative probabilities of hyperfragment production predicted by a statistical decay model [14] for a primary ${ }_{\Lambda}^{9} \mathrm{Li}$ nucleus as a function of its excitation energy. We also included in these calculations the recently observed ${ }_{\Lambda}^{6} \mathrm{H}$ hypernucleus [15] and a possible bound $\mathrm{n} \Lambda$ system (see discussion in [16]).

\begin{tabular}{|c|c|c|c|c|c|c|}
\hline Nucleus & $\mathrm{B}_{\Lambda}[\mathrm{MeV}]$ & & Nucleus & $\mathrm{B}_{\Lambda}[\mathrm{MeV}]$ & & $\Delta \mathrm{B}_{\Lambda}[\mathrm{MeV}]$ \\
\hline${ }_{\Lambda}^{4} \mathrm{H}$ & $2.04 \pm 0.04$ & [27] & ${ }_{\Lambda}^{4} \mathrm{He}$ & $2.39 \pm 0.03$ & [27] & $0.35 \pm 0.05$ \\
\hline${ }_{\Lambda}^{6} \mathrm{He}$ & $4.18 \pm 0.10$ & [27] & ${ }_{\Lambda}^{6} \mathrm{Li}$ & $5.89 \pm 0.37$ & {$[31]$} & $1.71 \pm 0.38$ \\
\hline${ }_{\Lambda}^{7} \mathrm{He}$ & $5.68 \pm 0.03 \pm 0.25$ & {$[26]$} & ${ }_{\Lambda}^{7} \mathrm{Be}$ & $5.16 \pm 0.08$ & {$[27]$} & $-0.44 \pm 0.09 \pm 0.25$ \\
\hline${ }_{\Lambda}^{8} \mathrm{Li}$ & $6.80 \pm 0.03$ & [27] & ${ }_{\Lambda}^{8} \mathrm{Be}$ & $6.84 \pm 0.05$ & [27] & $0.04 \pm 0.06$ \\
\hline${ }_{\Lambda}^{9} \mathrm{Li}$ & $8.50 \pm 0.12$ & {$[32]$} & ${ }_{\Lambda}^{9} \mathrm{~B}$ & $8.29 \pm 0.18$ & {$[32]$} & $-0.21 \pm 0.22$ \\
\hline${ }_{\Lambda}^{10} \mathrm{Be}$ & $9.11 \pm 0.22$ & {$[27]$} & ${ }_{\Lambda}^{10} \mathrm{~B}$ & $8.89 \pm 0.12$ & {$[27]$} & $-0.22 \pm 0.25$ \\
\hline${ }_{\Lambda}^{12} \mathrm{~B}$ & $11.37 \pm 0.06,11.52 \pm 0.35$ & {$[27][25]$} & ${ }_{\Lambda}^{12} \mathrm{C}$ & $10.76 \pm 0.19$ & {$[27]$} & $-0.61 \pm 0.20,-0.76 \pm 0.40$ \\
\hline
\end{tabular}

Table 1: $\Lambda$ separation energies for mirror hypernuclei with $\mathrm{A} \leq 12$. Only in the case of ${ }_{\Lambda}^{7} \mathrm{He}$ [26] systematic errors are explicitly quoted.

serve as a solid ground to link the basic long range nucleon-nucleon interaction to spectroscopic information of bound nuclei or NN scattering data.

On the long term Lattice QCD promises to revolutionize not only our understanding of isolated hadrons but also our picture of multi-hadron systems and nuclei. First calculations of nucleonnucleon and nucleon-hyperon interactions [17, 18] and explorative studies of light nuclei [19] and hypernuclei [20] were already performed. Eventually they may provide stringent boundary conditions for baryon-baryon potentials and will offer the extension into the regime of small distances which is mandatory to describe neutron star matter. Clearly, also these calculations need guidance by experimental data.

Ground state masses provide crucial benchmarks for any nuclear structure calculation. Like in conventional nuclei, where e.g. charge symmetry breaking is visible in binding energy differences of mirror-nuclei [21], also mirror-hypernuclei are extremely important to explore the isospin dependence of hyperon-nucleon interactions (see e.g. [22, 23]). Since the effects of multi-baryon 
forces or charge symmetry breaking are expected to be small in nuclei, high precision data on several light hypernuclei are of vital importance for the unfolding and accurate determination of the missing parts of interactions among the baryons of the fundamental SU(3) flavor octet. As can be seen from Tab. 1 the present experimental situation on mirror hypernuclei is - even ignoring possible systematic uncertainties - far from being satisfactory.

Employing quasi two-body kinematics, ground (and excited) hypernuclear states can be identified by a missing-mass analysis of the incident beam and the observed associated meson (left part of Fig. 1). Because these reactions require stable target nuclei, the hypernuclei accessible by these reactions are limited and all lie close to the valley of stability. In fact not a single pair of light mirror nuclei can be produced using the same projectile i.e. the same reaction process [24]. Unfortunately, a comparison of ground state masses from different reaction channels introduces large systematic errors in the range of typically a few hundred $\mathrm{keV}$ (see e.g. Refs. [25, 26] and Tab. 1). On the other hand, hypernuclei can be formed as secondary particles emerging from more or less violent nuclear interactions. The formation of a hypernucleus is usually tagged by its delayed weak decay producing a secondary vertex. Spectroscopic information is obtained exclusively by analysing the decay products. Groundstate binding energies can then be derived from the observation of the non-mesonic or the mesonic weak decays.

The production of a highly excited primary hypernucleus in an energetic nuclear reaction and its subsequent decay gives access to a variety of light and exotic hypernuclei (left part of Fig. 2), some of which cannot be produced or measured precisely by other means. Indeed, during the first two decades of hypernucleus research, weak hypernuclear decays observed in nuclear emulsions were the main source of information on groundstate binding energies. Even today, emulsion data (for a summary see [27]) provide for many nuclei the most precise value for the $\Lambda$-binding energies (see Tab. 1). The monoenergetic pions produced in the weak mesonic two-body decay of hypernuclei at rest are in principle the most direct measure of $\Lambda$ binding energies. However, despite extensive calibration measurements, uncertainties in the range-energy relation and in the emulsion density cause residual systematic uncertainties in the $\Lambda$-binding energies of at least 50 $\mathrm{keV}$ [28]. In case of the pionic two-body decay of ${ }_{\Lambda}^{4} H \rightarrow \pi+{ }^{4} \mathrm{He}$ this uncertainty may even be larger [29, 30]. More recently decay pion spectroscopy has been successfully performed at KEK [33] and by the FINUDA Collaboration at the DA $\Phi N E \mathrm{e}^{+} \mathrm{e}^{-}$collider in electronic experiments. Nevertheless, the momentum resolution and hence the absolute mass determination was limited in these mesurements [34].

\section{Decay pion spectroscopy at MAMI}

Following the first results presented from the KEK experiment [33], the usage of magnetic spectrometers to measure the momenta of decay pions from electroproduced hypernuclei with high resolution was proposed in 2007 for the Jefferson Lab [35]. This method combines the large production yield of the electroproduction of hypernuclei with the high momentum resolution of magnetic spectrometers. It opens the possibility to measure ground state masses of a variety of nuclei off the valley of stability with hitherto unrivaled precision within the same experiment and thus with small systematic uncertainties. Like in the previous pionic decay studies this method requires that the hypernucleus is at rest at the time of its decay. By detecting produced kaons, reactions 


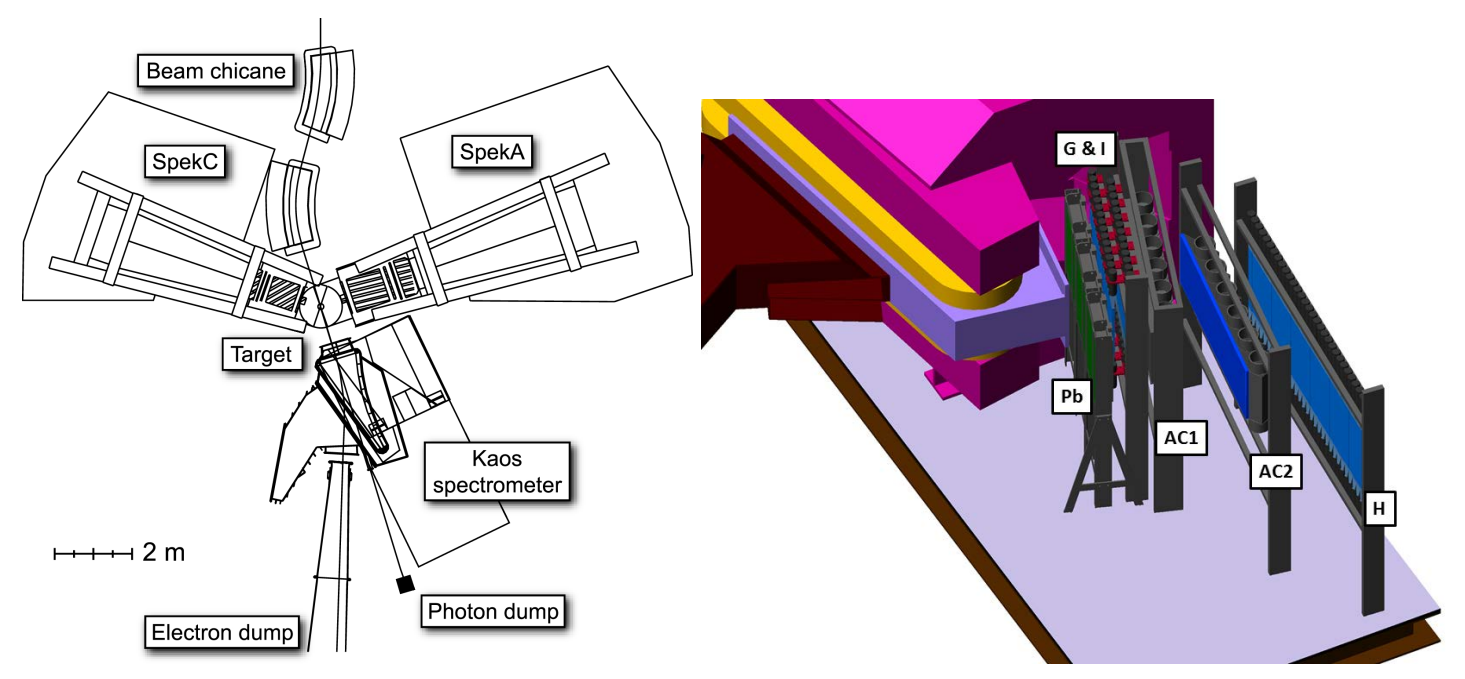

Figure 3: Left part: Arrangement of the spectrometers for the decay pion spectroscopy experiment. In this figure the electron beam enters from the top. The KAOS spectrometer used as a kaon tagger is placed at a $0^{\circ}$ scattering angle. The decay pions can be detected either in SpekA placed at an angle of $90^{\circ}$ with respect to the beam direction at the target or in SpekC at an angle of $126^{\circ}$. In order to guide the electron beam onto the existing beam dump two chicane magnets deflect the primary beam upstream of the target. Right: Detector setup of KAOS in the 2012 experiment. A 10-14 cm thick lead wall ( $\mathrm{Pb})$ was added with respect to the 2011 pilot experiment to absorb positron background. Furthermore a third time-of-flight wall (I) was added and a second aerogel Cherenkov detector (AC2) was added to improve the particle identification of the kaon tagger.

involving strangeness production can be tagged. Eventually the associated $\Lambda$ hyperon is captured by the remaining nucleus and forms a highly excited hypernucleus. Due to its high excitation this primary hypernucleus is likely to fragment into a lighter hyperfragment and one or more nucleons or light nuclei. While missing mass experiments are limited to nuclei close to the initial target, the quasi-free kaon production of an excited primary hypernucleus - e.g. ${ }^{9} \mathrm{Be}\left(e, e^{\prime} K^{+}\right){ }_{\Lambda}^{9} \mathrm{Li}^{*}-$ and its subsequent decay gives access to a variety of light and exotic hypernuclei, some of which cannot be produced or measured precisely by other means (left part of Fig. 2). To illustrate the scope in these reactions the right part of Fig. 2 shows the relative yield of hypernuclei predicted by a statistical decay model [14] for an initial ${ }_{\Lambda}^{9} \mathrm{Li}^{*}$ nucleus as a function of its excitation energy.

The electron accelerator MAMI consists of three stages of racetrack microtrons and one stage in the form of a double-sided harmonic microtron [36]. It can deliver a continuous wave electron beam with energies in the range of $180 \mathrm{MeV}$ to $1.6 \mathrm{GeV}$ and currents of up to $100 \mu \mathrm{A}$.

At present, the A1 spectrometer hall is one of the two major experimental facilities at MAMI. Three high resolution magnetic spectrometers are operated which can be positioned around solid state, liquid or high pressure gas targets at variable angles [37]. A key feature of these spectrometers is their high relative momentum resolution in combination with relatively large angular and momentum acceptances (Tab. 2). With the KAOS spectrometer the A1 spectrometer hall was extended by a short orbit spectrometer dedicated to the detection of charged kaons which serves as a kaon tagger in this experiment [38]. Its single dipole configuration allows to place the spectrometer at a $0^{\circ}$ position in beam direction, where the kaon cross section for hypernuclei production is 


\begin{tabular}{lccc}
\hline & SpekA & SpekC & KAOS \\
\hline \hline central momentum $[\mathrm{MeV} / \mathrm{c}]$ & 115 & 125 & 924 \\
central angle wrt. beam [deg] & 90 & 126 & 0 \\
momentum acceptance [\%] & 20 & 25 & 50 \\
solid angle acceptance [msr] & 28 & 28 & 12 \\
dispersive angle coverage [mrad] & \pm 70 & \pm 70 & \pm 185 \\
non-dispersive angle coverage [mrad] & \pm 100 & \pm 100 & \pm 20 \\
length of central trajectory to ToF-wall H [m] & 10.75 & 8.53 & 6.2 \\
first order relative momentum resolution & $<10^{-4}$ & $<10^{-4}$ & $\sim 10^{-3}$ \\
\hline
\end{tabular}

Table 2: Important parameters of the experimental set-up at MAMI. As high precision pion spectrometers, SpekA and SpekC are used. The KAOS spectrometer serves as a kaon tagger and therefore requires a large momentum acceptance at angles close to $0^{\circ}$.

expected to be large.

The arrangement of the spectrometers and the beamline is shown in the left part of Fig. 3. At $0^{\circ}$ the electron beam is guided through the magnetic field of the KAOS spectrometer after impinging on the target. In order to steer the beam onto the existing beam dump, the primary electron beam has to enter KAOS with a non-zero angle Therefore, it is directed onto the target with a predefined angle by two chicane magnets in the beamline upstream of the target. This angle is defined by the magnetic field strength of the KAOS magnet. For the given field strength of $1.2 \mathrm{~T}$ in the present experiment, the beam angle was set to $17^{\circ}$. Furthermore a secondary beam dump was built to capture bremsstrahlung photons.

Positively charged particles are then deflected into a detector assembly consisting of two timeof-flight walls with an aerogel threshold Cherenkov detector in between. For the 2012 run a third time-of-flight wall and a second aerogel Cherenkov detector were added to improve the particle identification of the kaon tagger.

In order to keep the background from bremsstrahlung low we focus for the production of light hyperfragments on low $\mathrm{Z}$ targets like ${ }^{12} \mathrm{C},{ }^{9} \mathrm{Be}$ and natural ${ }^{6,7} \mathrm{Li}$. In the 2011 and 2012 experiments a $22 \mathrm{mg} / \mathrm{cm}^{2}$ thick beryllium foil was bombarded by a $1.5 \mathrm{GeV}$ electron beam. According to the statistical decay calculations [14] this target thickness should be sufficient to stop all hyperfragments except for the light ${ }_{\Lambda}^{3} \mathrm{H}$ and ${ }_{\Lambda}^{4} \mathrm{H}$ which are only partially stopped. Even though the stopping probability for these light hyperfragments would increase with increasing target thickness, the larger energy loss variation for the decay pions would lead to wider peaks in the pion spectrum and therefore a lower signal to background ratio. To reduce the energy loss variation even further, the target foil was rotated by $54^{\circ}$ with respect to the beam, thus minimizing the path length of the pion inside the target.

\section{Status of data analysis}

The main difficulty in the analysis of the data acquired in the 2011 pilot experiment was caused by the high flux of background particles in the KAOS spectrometer which leads to a large random coincidence probability. This background is dominated by positrons produced by the conversion of 

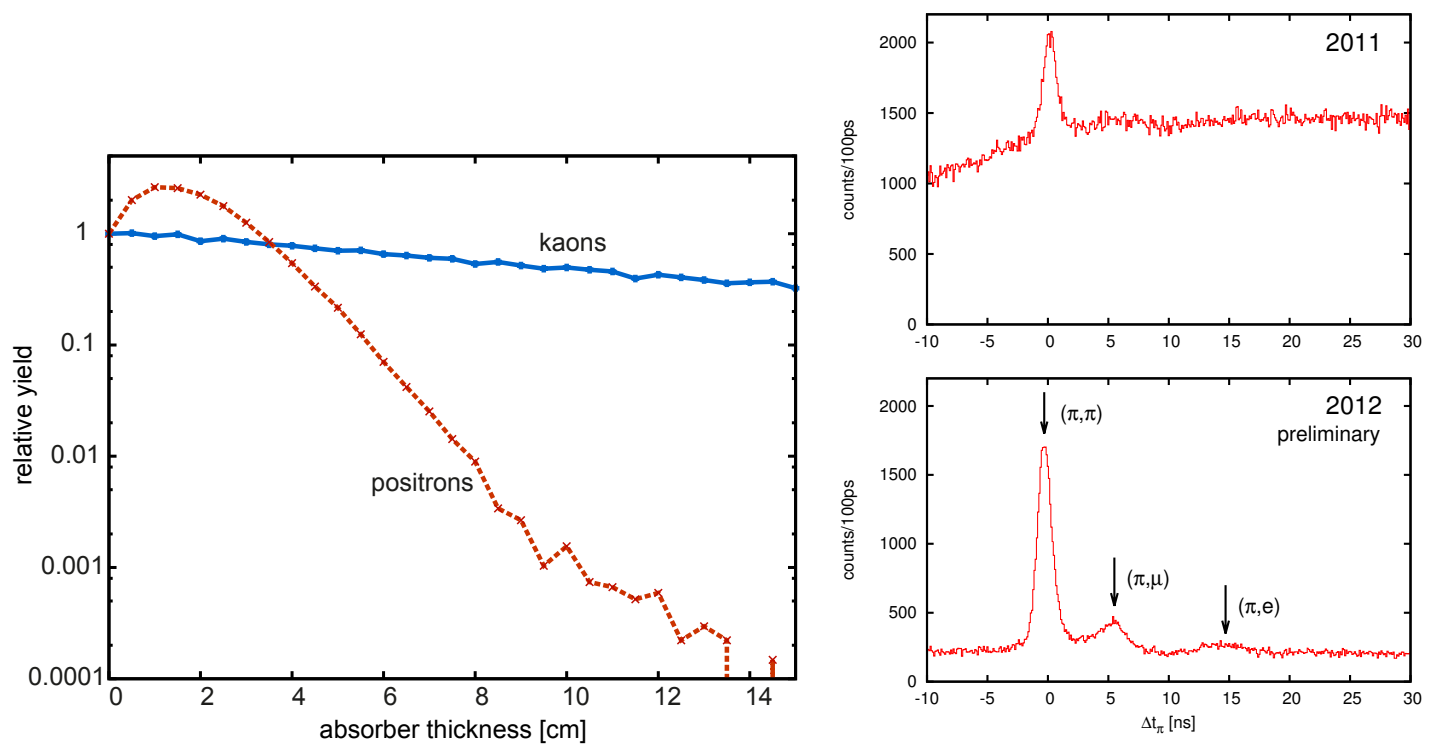

Figure 4: Left part: Relative yield of positrons and kaons behind a lead absorber in KAOS as a function of its thickness. Right: Measured coincidence time between the kaon tagger and one of the high resolution spectrometers corrected for the hypothesis of pion-pion coincidences from the 2011 pilot run (top) and the experiment performed in October 2012. In the 2011 data the background from random pion-positron events dominates the spectrum.

bremsstrahlung photons in the target, but also pions and protons are detected in the KAOS spectrometer. To separate kaons from the background of other particles, several observables are accessible in the spectrometer data: the time-of-flight between different scintillator planes within KAOS , the energy-loss in the scintillator walls, the aerogel Cherenkov information, and the out-of-plane angle of the particle. The latter was used in the analysis of the 2011 data, since the positron background peaks in the bending plane. Once a particle has been identified in KAOS , the effective time-offlight relative to the particle detected in one of the high resolution spectrometers allows to identify specific particle pair combinations.

Within the momentum range of KAOS, protons can be cleanly discriminated from the background of positrons and pions. Even though pions (as well as kaons) cannot be separated from the positron background by using the KAOS spectrometer information alone, pion-pion coincidences between one of the high resolution spectrometers and KAOS can be identified in the coincidence time spectrum - albeit with large background - as it is shown in the top right part of figure 4. Here the effective time-of-flight $\Delta t_{\pi}$ takes the measured momenta of the coincident particles into account and assumes a coincident $\pi^{+}-\pi^{-}$pair. Pions detected in KAOS and muons from pion decays close to the target entering the high resolution spectrometers show up in Fig. 4 as a peak shifted by about $5 \mathrm{~ns}$.

Unfortunately the yield of kaons compared to the background is so small, that in the 2011 pilot experiment it was not possible to retrieve a clean kaon sample. A larger luminosity by simply increasing the beam intensity or the target thickness would of course lead to a larger kaon yield. However, this would at the same time cause an over-proportional increase of the number of random positron-pion coincidences. Thus the background from random positron-pion coincidences can 
only be reduced by eliminating most of the positrons from the experimental acceptance. This is possible by blocking positrons by means of a lead absorber in front of the detector system of the KAOS spectrometer. A 10-14 cm thick lead wall reduces the number of positrons by more than 3 orders of magnitude but still enables around $40 \%$ of the kaons (and pions) to enter the KAOS detector system. The successful reduction of the background in the 2012 experiment is illustrated in the right lower part of Fig. 4. Compared to the 2011 run the signal-to-background ratio improves by an order of magnitude. The additional bump which is now visible around $\Delta \mathrm{t}_{\pi} \approx 15 \mathrm{~ns}$ is caused by real pion-electron coincidences. Since the tracking code and the particle identification software package need to be adapted to the modified detector geometry it is in the present stage of the analysis not possible to make more quantitative statements. Nevertheless, due to the increased beam intensity from about $2 \mu \mathrm{A}$ to $20 \mu \mathrm{A}$, the reduced deadtime and the longer running period - we expect a significantly larger kaon yield compared to the 2011 pilot run as already indicated in the preliminary analysis shown in the right part of Fig. 4. Another rise of the beam intensity and the usage of a stack of several thin separated and tilted targets will in future studies allow to increase the luminosity even further without sacrificing the excellent pion momentum resolution.

\section{Acknowledgments}

Work supported in part by the Federal State of Rhineland-Palatinate and by the Deutsche Forschungsgemeinschaft (DFG) with the Collaborative Research Center 1044, by the Research Center 'Elementary Forces and Mathematical Foundations' (EMG), and by the Carl Zeiss Foundation. We also acknowledge the support by the Research Infrastructure Integrating Activity 'Study of Strongly Interacting Matter' HadronPhysics3 under the 7th Framework Programme of EU. The Japanese group of the collaboration acknowledges the support by Strategic Young Researcher Overseas Visits Program for Accelerating Brain Circulation (R2201) and support by the Core-to-Core program of Japan Society for Promotion of Science (21002).

\section{References}

[1] P.B. Demorest et al., Nature 467, 1081 (2010).

[2] W. Weise, Lectures at the Erice School 2011 "From Quarks and Gluons to Hadrons and Nuclei", arXiv:1201.0950 [nucl-th].

[3] K. Masuda, T. Hatsuda, T. Takatsuka, arXiv:1205.3621 [nucl-th].

[4] S. Weissenborn, D. Chatterjee, J. Schaffner-Bielich, Nuclear Physics A 881, 62 (2012).

[5] R. Lastowiecki, D. Blaschke, H. Grigorian, S. Typel, Acta Phys. Pol. B Proc. Suppl. 5, 535 (2012).

[6] D.L. Whittenbury, J.D. Carroll, A.W. Thomas, K. Tsushima, J.R. Stone, arXiv:1204.2614 [nucl-th].

[7] Wei-Zhou Jiang, Bao-An Li, Lie-Wen Chen, The Astrophysical Journal 756, 56 (2012).

[8] T. Katayama, T. Miyatsu, K. Saito, ApJ Supplement Series 203, 22 (2012).

[9] K. Kiuchi, Y. Sekiguchi, K. Kyutoku, M. Shibata, Class. Quantum Grav. 29, 124003 (2012).

[10] Lee Lindblom and Benjamin J. Owen, Phys. Rev. D 65, 063006 (2002).

[11] N. Andersson, K.D. Kokkotas, Int. J. Mod. Phys. D 10, 381 (2001). 
[12] M.G. Alford, S. Mahmoodifar and K. Schwenzer, Phys. Rev. D 85, 044051 (2012).

[13] H.-W. Hammer, A. Nogga, A. Schwenk, Rev. Mod. Phys. 85, 197 (2013).

[14] A. Sanchez Lorente, A.S. Botvina, and J. Pochodzalla, Phys. Lett. B 697, 222 (2011).

[15] M. Agnello et al., Phys. Rev. Lett. 108, 042501 (2012).

[16] A.S. Botvina, I.N. Mishustin, and J. Pochodzalla, Phys. Rev. C 86, 011601(R) (2012).

[17] N. Ishii [PACS-CS and HAL QCD Collaborations], arXiv:1004.0405v1 [hep-lat].

[18] S. Aoki et al., [HAL QCD Collaboration], arXiv:1206.5088 [hep-lat].

[19] T. Yamazaki [PACS-CS Collaboration], PoS Lattice2010:021,2010, arXiv:1012.0410v1 [hep-lat].

[20] S.R. Beane et al., arXiv:1206.5219 [hep-lat].

[21] G.A. Miller, A.K. Opper and E.J. Stephenson, Annu. Rev. Nucl. Part. Sci. 56, 253 (2006) and references therein.

[22] A. Nogga, H. Kamada, and W. Glöckle, Phys. Rev. Lett. 88, 172501-1 (2002).

[23] E. Hiyama et al., Phys. Rev. C 80, 054321 (2009).

[24] J. Pochodzalla, Acta Phys.Polon.B42, 833-842 (2011).

[25] L. Yuan et al. [HNSS Collaboration], Phys. Rev. C 73, 044607 (2006).

[26] S.N. Nakamura et al. [HKS (JLab E01-011) Collaboration], Phys. Rev. Lett. 110, 012502 (2013).

[27] D.H. Davies and J. Pniewski, Contemp. Phys.27 91 (1986) and refernces therein.

[28] M. Juric et al., Nucl. Phys. B52, 1 (1973).

[29] G. Bohm et al., Nucl. Phys. B4, 511 (1968).

[30] G. Bohm et al., Nuovo Cim. 70, 384 (1970).

[31] D.-M. Harmsen, Phys. Rev. Lett. 19, 1186 (1967).

[32] J. Pniewski et al., Nucl. Phys. A443, 685 (1985).

[33] H. Tamura et al., Phys. Rev. C 40, R479 (1989).

[34] M. Agnello et al. [FINUDA Collaboration], Phys. Lett. B 681, 139 (2009).

[35] L. Tang et al., Exp. Proposal E-08-012, Jefferson Lab (2007).

[36] K.-H. Kaiser et al., Nucl. Instr. Meth. Phys. Res. A 593, 159 (2008).

[37] K.I. Blomqvist et al., [A1 Collaboration], Nucl. Instr. Meth. Phys. Res. A 403, 263 (1998).

[38] P. Achenbach [A1 Collaboration], Eur. Phys. J. ST 198, 307 (2011). 